Anuario Latinoamericano

Ciencias Políticas

y Relaciones Internacionales

vol. 4, 2017

pp. $41-60$

\section{El campo de los estudios de la violencia en México}

DOI: $10.17951 /$ al.2017.4.41

\section{Field of study on violence in Mexico}

\author{
José Alfredo Zavaleta Betancourt ${ }^{1}$ \\ UNIVERSIDAD VERACRUZANA, \\ XALAPA, MÉXICO \\ zavaletabetancourt@gmail.com
}

\section{RESUMEN}

El presente trabajo analiza la lógica del campo de estudios de la violencia y el delito en México a partir del contexto de inseguridad, las luchas discursivas entre diferentes agentes universitarios, gubernamentales $y$ social-civilistas $y$ algunos programas de investigación que en las últimas tres décadas han analizado los principales factores de la violencia social. Para tal efecto, propone observar la violencia y el delito como un campo para no reducir la lógica de estos procesos a la tasa de homicidios; analiza los logros de programas de investigación dominantes en algunos trabajos sobre el tema producidos en la historia, la sociología, la ciencia política y la antropología mexicana contemporánea para luego señalar algunos de los retos de la agenda de las ciencias sociales en los estudios de estos procesos. El trabajo cierra con la propuesta de consolidación de algunas líneas de investigación que han permitido logros científicos que pueden ser considerados patrimonio en el campo, tanto como ofrece algunos elementos teóricos e institucionales para el desarrollo de la agenda de investigación para los próximos años.

PALABRAS CLAVE: violencia, delito, campo de estudios de la violencia y el delito, impunidad.

\section{ABSTRACT}

This paper analyzes the logic of the field of study on violence and crime in Mexico in the context of insecurity, discursive struggles between different university, government and social-civilist agents and some research programs which analyzed the main factors of social violence in the last three decades. To this end, it proposes to observe violence and crime as a field of study instead of reducing the logic of these processes

1 Doctor en sociología, investigador del Instituto de Investigaciones Histórico Sociales, Sistema Nacional de Investigadores, México, Nivel I, e integrante del Grupo de Trabajo: "Paradojas de la Seguridad Ciudadana en América Latina del Consejo Latinoamericano de Ciencias Sociales". 
Dossier América Latina: violencia en México y Centroamérica to the homicide rate. It analyzes the achievements of some research programs on the subject produced in history, sociology, political science and contemporary Mexican anthropology and then points out some of the challenges that social sciences face in the study of these processes. The work closes with the proposal of consolidation of some lines of research that have allowed scientific achievements and can be considered heritage in the field. It also provides some theoretical and institutional elements for the development of the research agenda for the coming years.

KEYWORDS: violence, crime, field of study on violence and crime, impunity.

\section{Presentación}

Para la reflexión situada de la violencia en nuestro país dejemos a los etnólogos el análisis de nuestra relación generacional con tales procesos. Dejemos a un lado, además, la confesión de que en algún momento de nuestra vida, practicamos conscientemente o inconscientemente la violencia, la deseamos, la sufrimos, la consideramos un instrumento de cambio -tal como Michel Foucault se interrogaba en algún momento acerca de si en el fondo la revolución fuese un sueño bárbaro (Henri-Lévy 1992)-, para reconocer que nuestro interés de conocimiento que busca la comprensión de la violencia nacional, si bien procede de nuestra experiencia generacional, no puede renunciar a las consecuencias prácticas de la observación en el presente.

La puesta entre paréntesis de nuestra relación con la violencia de los años recientes sólo importa, por ahora, como indicio de nuestro posicionamiento y agencia en el campo de la violencia y el delito. Es posible que nuestros discursos varíen no sólo con base en nuestras experiencias y prácticas de victimización directa o indirecta, sino que dependan de nuestras trayectorias académicas. Desde esta perspectiva, podemos aceptar que la distancia teórica que supone la objetivación de la violencia habla en efecto de nosotros, pero al mismo tiempo, requiere de no confundir nuestras experiencias violentas, como víctimas o victimarios, con las experiencias de los otros, aún cuando sean de nuestro grupo social o clase.

En la medida en que no hay registro nacional legítimo, si se generaliza un caso local como nacional, ni se ejerce la violencia de la misma forma en todas las regiones -como sostienen para el caso colombiano José Darío Rodríguez Cuadros (2015) y Fernán Enrique González y González (2015) - todo análisis regional es parroquial. Así, cualquiera que sea la región del país desde la cual observamos parcialmente estos procesos, puede advertirse que la violencia y el delito aparecen entre nosotros, aún en muchos casos, como objetos "parciales" e ilegítimos, ligados a la maldad, respecto de los cuales o decidimos por voluntad no hacer investigación o de los cuales por riesgo o peligro tenemos que guardar silencio.

En efecto, la fuerza de la violencia social nos ha obligado a comprenderla generacionalmente tal como Max Weber (1988) se interrogaba acerca de 
cómo proceder acerca de la comprensión de "las particularidades [...] el elemento individual y peculiar de un contexto" en la búsqueda de la causalidad. En los años recientes de reformas neoliberales, la historia, la sociología y la antropología mexicana -a veces más inclinadas a los discursos anglosajones que a los latinoamericanos- hemos construido un campo de estudios sobre la violencia y el delito que ha logrado sobreponerse al descrédito de los discursos de la violencia revolucionaria.

No ha sido fácil. La observación sistemática de la violencia y el delito es hasta ahora, -a pesar de la centralidad que ahora ocupa en las ciencias sociales mexicanas-, un tema con el cual no se sienten a gusto quienes consideran a la violencia como ligada a las categorías sociales en descomposición, un instrumento de emancipación política o que estos asuntos aún pertenecen al universo simbólico de la policía. En un país semi-periférico como el nuestro es comprensible tal cautela. La violencia política y social ha sido frecuente en nuestra historia contemporánea y se relaciona con el autoritarismo político, las cosas de la policía, como una matriz de abusos y excepciones; sin embargo, estas aproximaciones pertenecen al tipo de discursos que transfieren la responsabilidad de la violencia a los "otros", se ubican en un afuera de ella, narran apocalípticamente "nuestro holocausto", o prolongan la narrativa del desencanto anti-político.

\section{Las luchas discursivas en el campo de estudios}

Por lo contrario a estas perspectivas, pueden registrarse en nuestro país múltiples trabajos de disciplinas diversas, historia, sociología, ciencia política y antropología, que en conjunto constituyen un campo de estudios sobre la violencia y el delito. La producción de este campo de estudios -cuyo referente es campo de la violencia y el delito- no ha sido un proceso libre de disputas. Este ha sido posible mediante crítica de la teoría de la transición y la consolidación democrática, la superación de los usos anti-políticos del socialcivilismo y reconocimiento en la observación del carácter productivo de la violencia legal e ilegal.

En efecto, el campo no ha sido un espacio neutral, por ejemplo, las corrientes teóricas que abordan este objeto no están de acuerdo acerca del sentido práctico del trabajo académico; por lo contrario, ha sido escenario de luchas discursivas, monopolios y estrategias. La lógica predominante en este campo -como dice Pierre Bourdieu (2005)- es la de "las cuotas de competencia de mercado". Desde las regiones centrales del campo, en su parcialidad, se describe a los procesos nacionales de violencia y delito mediante estrategias de generalización de procesos regionales heterogéneos que en muchos casos son la ampliación del sentido local que tienen los procesos metropolitanos. Por lo contrario, los análisis regionales frecuentemente adolecen de perspectivas globales y ambición teórica para englobar lo nacional de tales procesos.
El campo de los estudios de la violencia en México José Alfredo Zavaleta Betancourt 
Dossier América Latina: violencia en México y Centroamérica
En las tres últimas décadas, estas luchas discursivas, a veces silenciosas, a veces públicas, han tenido como puntos de desacuerdo la primacía de la práctica sobre los discursos teóricos, la pertinencia de los discursos del derecho y la criminología de países centrales para el análisis de los procesos de violencia en un país semi-periférico como México, la metodología de investigación empírica correcta, las pretensiones de validez de las observaciones parroquiales, la gestión privada y gubernamental de los sistemas de información pública sobre delitos, inseguridad y victimización.

Es paradójico, pero la construcción del campo de estudios de estos procesos ha tenido como prejuicio la idea moral de la violencia sociopolítica como una variable negativa de nuestra vida social porque nos ha impedido el reconocimiento de que nuestra sociedad ha sido construida a partir de innumerables acontecimientos violentos. Por supuesto, es deseable que las reformas a las que aspiramos no sean anómicas aunque hayamos aprendido que es altamente improbable que las reformas de las instituciones de seguridad y justicia a las que aspiramos sean de terciopelo.

Desde el punto de vista organizacional, el campo de estudios de la violencia y el delito está constituido por un conjunto de relaciones discursivas reproducidas mediante redes académicas de cuyos temas y datos eventualmente técnicos y funcionarios toman información para la retórica de sus políticas y programas. El campo funciona como una reflexión crítica que irrita al sistema político, particularmente a sus instituciones de seguridad y justicia.

En estas luchas participan los investigadores metropolitanos y regionales que disputan el prestigio en el campo, instituciones de inteligencia militar y policíaca, organizaciones civiles autónomas del sistema de seguridad o las incentivadas por aquél, consultorías que participan de las disputas de recursos económicos para el análisis de los factores de la violencia y el delito, observatorios institucionales y empresariales, medios de comunicación y periodistas que en algunas regiones del país enfrentan el peligro de las extensiones autoritarias de los estados en los cuales no ha gobernado nunca un partido diferente del viejo régimen.

En ocasiones olvidamos que las ciencias sociales no sólo se producen en las universidades, también se adaptan, producen y reproducen en diferentes tipos de organizaciones e instituciones que con frecuencia rechazan la información producida por investigadores universitarios bajo la retórica de la denuncia del teoricismo, la autoreferencia, el periodismo presuntuoso, la historia de las ideas de especialistas. Es necesario tomarse con toda seriedad el siguiente reproche:

La segunda tentación a evitar es la que a veces nos da a los académicos y a los analistas del tema: la autoreferencia. Es la tentación que a veces tenemos de enfocarnos en lo técnico y discutirlo sólo entre nosotros, citarnos en nuestros documentos y pensar que así logramos un impacto. La autoreferencia es no abrirnos más a la sociedad, no comunicarnos mejor con la gente, con las organizaciones locales, con las personas que están en el terreno. El rigor académico y analítico es necesario e indispensable para una política pública. Pero no es suficiente. Tene- 
mos que crear comunidades de conocimiento más abiertas y más amplias. Si no, seguiremos predicándole sólo al coro de la iglesia (Jaime 2016).

Asimismo:

Las ciencias sociales no nos proveen lo suficiente para entender las formas en que el crimen influye sobre un sistema de gobierno democrático... carecemos de estudios adecuados acerca de los efectos políticos de la criminalidad -traducida en actos generalizados, que se esparcen a lo largo del territorio, o en empresas organizadas, ya sean violentas o no-con relación a la sociedad civil, el régimen y el estado (Bailey 2014: 11).

De una matriz discursiva similar procede el síntoma de que la violencia en el país ha sido sobre-diagnosticada. Es posible que esta percepción sea el resultado del interés en la gobernabilidad del crimen o del desencanto ante la alta improbabilidad de las reformas de las instituciones de seguridad y justicia decretadas en los años recientes. En este síntoma se basa la estrategia discursiva gubernamental de pacificación, para la continuación de la guerra contra la delincuencia organizada por otros medios o bien para incentivar la implementación de las reformas policial y de justicia penal en los estados.

En realidad sabemos aún muy poco acerca de la violencia social de las últimas tres décadas porque, o bien la observamos como un tipo de violencia política que amenaza el viejo régimen, por ejemplo, bajo argumento que socava el monopolio de la violencia estatal, o bien la observamos negativamente como una patología que desestructura las relaciones sociales en la familia, la escuela, el barrio o el municipio. Decía Samuel González Rodríguez:

Que ya tenemos un punto de saturación que es importante. Como analista me puedo acordar de todos los hechos de violencia que surgieron entre 1988 y 1997, puedo recordarlos porque son 20, 30 ó 35, sin embargo, no soy capaz de recordar lo que pasó en México en los últimos 45 días donde un acontecimiento es peor que el otro. Por ejemplo, ya no nos acordamos que el 15 de septiembre del 2008 tiraron una bomba durante la celebración de la independencia de México en Michoacán, y eso se debe a que el torbellino y la aceleración es tal que la saturación nos llega en todos aspectos (González Rodríguez 2011).

En verdad, aún sabemos muy poco acerca de la continuidad y discontinuidad de la violencia caciquil -de los años setenta y ochenta en las regiones del país-, la violencia contrainsurgente y la violencia social; conocemos poco acerca de la articulación y predominancia de estas modalidades de violencia en los espacios rurales y urbanos; poco también sobre cómo los delitos alimentan la violencia, o lo contrario.

Aún no objetivamos satisfactoriamente los tipos de violencia en las escuelas que anuncia el bullying, las inflexiones de las trayectorias sociales de
El campo de los estudios de la violencia en México

José Alfredo Zavaleta Betancourt 
Dossier América Latina: violencia en México y Centroamérica jóvenes que deciden incorporarse al sub-campo de la violencia organizada; sabemos poco, -más allá de las ficciones de capturas invertidas o integrales, de algunos analistas-, acerca de la corrupción institucional avanzada que se reproduce detrás de los abusos policiales y militares, o bien de las interfaces de la corrupción administrativa e institucional; poco sobre los mecanismos de lavado de dinero producto del tráfico de drogas, extorsiones y secuestros; poco acerca de las implicaciones para la ciudadanía regional del incremento de la letalidad del uso de la fuerza de las agencias estatales o muy poco sobre la naturaleza de la guerra que se libra en nuestro país.

Evidentemente, sabemos de estas problemáticas por la prensa (Martínez 2010; Lemus 2015) que -a propósito de otros países en los cuales llegó a considerarse un obstáculo para la sociología- entre nosotros es un insumo imprescindible para la objetivación de la violencia en muchas regiones del país donde es imposible el trabajo de terreno; asimismo, sabemos algo de ellas por los analistas que denuncian un supuesto colapso estatal mediante las metáforas de instituciones fallidas, narco-insurgencias (Buscaglia 2013).

Por supuesto sabemos algo acerca de estos procesos a partir de investigaciones académicas y científicas que interpretan con modelos anglosajones procesos regionales. Hemos acumulado información cuantitativa y cualitativa en tesis universitarias, ensayos, libros e informes técnicos (Arteaga Botello y Arzuaga Magnoni 2016) acerca de la nueva morfología de la violencia social, pero no tenemos acuerdos acerca de si en nuestro trabajo tiene primacía la prueba de teorías con pretensiones universales o la reconstrucción articulada de procesos regionales mediante el uso crítico de la teoría.

En síntesis, aún no sabemos lo suficiente para el diseño de políticas basadas en evidencia; por lo contrario, persisten algunos enigmas tales como: la especificidad de la relación entre el sentimiento de inseguridad, la violencia y los delitos; los perfiles de victimarios; las trayectorias sociales de los desaparecidos; el desplazamiento forzado por la delincuencia organizada en el contexto de las concesiones al neoextractivismo; los enclaves de robo de combustible, narcofosas y los sujetos desechados; el suicidio que ya no se investiga; el intercambio de recursos y funciones entre redes delictivas, políticos y militares y policías; la gobernabilidad de los operativos conjuntos, la gestión política de las respuestas a las solicitudes de información que abren y cierran datos según coyunturas políticas y electorales (RGVS 2015).

\section{Los programas de investigación dominantes en el campo}

El uso de la teoría en el campo de la violencia y el delito puede orientarse por el reconocimiento del carácter situado de los discursos y la necesidad del sentido práctico, mediante la reflexión de las consecuencias del trabajo académico.

Para la observación de los procesos de violencia social, las ciencias sociales mexicanas no deberían desarrollar como elemento principal de la agenda de 
investigación la divulgación de tal o cual autor estelar de las disciplinas de los países centrales. El retorno a los clásicos o los comentarios acerca de los autores de las sociedades centrales tiene una función en nuestro campo más o menos de recuerdo eventual del patrimonio metodológico o el imaginario de la integración universal, pero nadie investiga ya como ellos lo hacían, ni se gana mucho con la demanda de interlocución en otras luchas discursivas en las cuales, en el mejor de los casos, somos aceptados como traductores.

La observación de la lógica de la violencia y el delito incluye el uso situado, local, reflexivo de las categorías de los procesos de violencia de los países centrales pero el olvido de la desigualdad histórica de los grupos sociales en nuestro país puede bloquear las posibilidades de observación de la singularidad de los procesos objetivados. Para la observación de éstos, la divulgación de las ideas es importante pero no suficiente. El análisis regional, situado que utiliza conceptos de teorías de sociedades centrales- no debe olvidar la historicidad de nuestras instituciones estatales y la desigualdad histórica que determinan los procesos de violencia. Dice F. E. González y González (2015):

[...] los nuevos estados fueron construidos sobre la base de poblaciones heterogéneas y a veces hostiles entre sí, a diferencia de Europa, donde la guerra debilitó las diferencias internas y homogeneizó a la población... se encuentran en una desigual y desventajosa posición dentro del sistema capitalista mundial [...] sobre todo [...] el Estado [...] es un actor político que no logra derrotar otras formas de poder social regional y otras redes segmentadas de poder, sino que se ve obligado a coexistir o competir con ellas por el control de la regulación política como un actor más, con más o menos poder y recursos (González y González 2015: 122-123).

El análisis regional de la violencia requiere de un posicionamiento académico para el cual no es suficiente la denuncia moral, el socialcivilismo liberal o, como decía Bourdieu, el "funcionalismo de lo peor".

El posicionamiento académico regional en el campo de los estudios de la violencia y el delito requiere del desanclaje reflexivo de algunos conceptos de las ciencias sociales europeas, sin embargo, el sentido práctico del uso de tales conceptos en investigaciones empíricas, regionales, situadas, evita que la reproducción de algunas teorías y conceptos, tales como "las ventanas rotas", la violencia como ausencia de conflicto, la seguridad ciudadana y la seguridad humana, sean simples frases mediante las cuales se legitiman prácticas estatales en los regímenes liberales o progresistas.

Las estrategias alternativas pueden tomar ejemplo de aquellos académicos reflexivos que, conscientes de la arbitrariedad de sus observaciones regionales o etnocéntricas, evalúan, por ejemplo, la teoría de las ventanas rotas para aceptar sus supuestos, pero para pensar otras posibilidades no represivas basadas en la hospitalidad y en la prevención (Roché 1996); interpretan los procesos de violencia en las ciudades para interrogarse acerca de la criminalización
El campo de los estudios de la violencia en México

José Alfredo Zavaleta Betancourt 
Dossier América Latina: violencia en México y Centroamérica y el encierro de los pobres que habitan los barrios (Wacquant 2001); critican los usos securitarios de la retórica de la seguridad ciudadana de gobiernos de derecha y progresista en los países periféricos y semi-periféricos latinoamericanos o analizan la posibilidad de una agenda social desde las organizaciones de la sociedad civil mediante el adjetivo desde abajo para la seguridad humana o el desanclaje y localización de las denominadas buenas prácticas de prevención de la violencia y el delito (Angarita Cañas 2015).

En estas circunstancias, quizá sea preciso señalar que en una sociedad históricamente violenta el estudio de la violencia no puede sino conceptuar a este proceso como un conjunto de prácticas de victimización mediante las cuales se daña el cuerpo y la dignidad de las personas. En efecto, las violencias son multidimensionales y multifactoriales y se configuran regionalmente de formas diversas, sobre todo en aquellos casos en los cuales la impunidad aparece como un mecanismo de reproducción. Respecto de la violencia en nuestro país Joaquín Villalobos (en: Maerker 2011) decía que le sorprendía que hubiéramos olvidado que somos violentos y tenemos un Estado débil:

¿Cómo es que tantos mexicanos se decidieron a matar? Buscando una respuesta a esta pregunta descubrí dos cosas sobre las que cambié de opinión con relación a este problema: una, creía que los mexicanos eran esencialmente pacíficos, pero hoy creo que no es así [...] yo creía que México tenía un estado fuerte, pero hoy percibo que tiene un estado grande pero no un estado fuerte (Villalobos en: Maerker 2011: 37).

La construcción de una respuesta distinta - sin el supuesto de una esencia nacional o la oposición simple débil-fuerte- mediante la observación de la violencia puede realizarse mediante el concepto de campo. La observación de la violencia y el delito como un campo evita la reducción de la densidad delictiva a la tasa de homicidios generalmente distinta de otros delitos de alto impacto. El campo de la violencia y el delito puede observarse desde las regiones como una red de relaciones locales reguladas por diversos regímenes de violencia mediante las cuales los victimarios — víctimas en otras circunstancias según la lógica de dominación en el campo_ producen la subjetividad de las víctimas en resistencia. La construcción de una teoría del campo de la violencia y el delito puede enfatizar la lucha entre agentes por la impunidad mediante reglas, estrategias, discursos, prácticas (Zavaleta Betancourt 2011).

La observación de los procesos de violencia en lo rural y lo urbano que se ha desarrollado en las tres últimas décadas en el país puede interpretarse como intervenciones en el campo para la reducción de la violencia y el delito a niveles tolerables. Respecto de este punto M. Foucault decía: "En términos generales la interrogación será, en el fondo, cómo mantener un tipo de criminalidad [...] dentro de límites que sean social y económicamente aceptables y alrededor de una media que se considere, para decirlo de algún modo, óptima para un funcionamiento social dado" (Foucault 2008). 
En retrospectiva, el campo de estudios en nuestro país ha experimentado tres ciclos caracterizados por el uso dominante de un programa de investigación en cada uno de ellos, por ejemplo, los análisis estadísticos acerca de la criminalidad de la capital del país, el antecedente más inmediato de los observatorios ciudadanos de seguridad y justicia; los discursos sobre la militarización y los derechos humanos, los antecedentes de los análisis sobre seguridad ciudadana y humana; y el análisis de la violencia en las ciudades del país, antecedente de las preocupaciones actuales acerca de la investigación etnográfica por la inter-legalidad, los performances del dolor y sufrimiento de las víctimas en los márgenes estatales.

Es posible que tales ciclos discursivos no sean evidentes debido a que algunos autores de trayectorias académicas hibridas han transitado por todos ellos, o bien porque en la actualidad la complejidad de la violencia en las regiones del país requiere del uso de estas perspectivas independientemente de las preferencias teóricas de los investigadores. Las luchas discursivas que se desarrollan actualmente en el campo de estudios sobre la violencia y el delito necesitan de una ponderación de estos ciclos y programas, la recuperación reflexiva del patrimonio que representan los acontecimientos discursivos que hicieron posible las actuales perspectivas dominantes: las encuestas de victimización e inseguridad, los investigadores de diversas universidades públicas metropolitanas y regionales y los trabajos de periodismo de investigación.

Estos acontecimientos pueden ayudarnos a entender como algunos investigadores dejaron de hablar de seguridad para advertir acerca de la necesidad de la observación de la violencia, cómo se institucionalizó gubernamentalmente el discurso de la seguridad ciudadana y se posicionó el tema de los márgenes estatales, la necropolítica (Maldonado 2010; Fuentes Díaz 2012; Sierra, Hernández, Sieder 2013), el estado de excepción (Das y Poole 2008), o bien se recuperó la idea hobbesiana de estado de guerra para la comprensión y explicación de los procesos de nuestra violencia (González Rodríguez 2014; Illades y Santiago 2014; Schedler 2015).

En esta evolución han tenido un papel significativo algunas luchas discursivas. Estos desacuerdos han permitido registros básicos del incremento de la inseguridad en el marco de las reformas neoliberales, el malestar público por la crueldad de la violencia social y la impunidad: la descripción de feminicidios, ejecuciones extrajudiciales, fosas clandestinas, corrupción de instituciones de seguridad y justicia; las fronteras de lo legal y lo ilegal, la vulnerabilidad de las víctimas, los vacíos de derecho e impunidad mientras se implementan, sin supervisión, hibridas, negociadas, las reformas policial y de justicia penal, como anotaremos más adelante, paralelas a la militarización de la seguridad y la de la prevención social de la violencia y el delito.

En las dos décadas pasadas, el campo de estudios experimentó acontecimientos que pueden considerarse un legado. Los debates más importantes en nuestro campo se abocan a la superación de las políticas de seguridad pública (Moloeznik 2005) mediante políticas de seguridad ciudadana en las ciudades
El campo de los estudios de la violencia en México

José Alfredo Zavaleta Betancourt 
Dossier América Latina: violencia en México y Centroamérica
(Rico y Chinchilla 2002) e incluso, con fuerte sentido normativo, de seguridad humana que consideran a la seguridad ciudadana como un elemento de aquella. Hay quienes hablan de seguridad humana desde abajo (Angarita Cañas 2015).

Este giro teórico no ha tenido hasta ahora influencia en las políticas públicas de los gobiernos neoliberales, quienes utilizan este discurso para la legitimación de viejas prácticas policiales y judiciales. Es similar lo que acontece, en otra correlación de fuerzas, con las políticas de seguridad ciudadana de los gobiernos latinoamericanos de izquierda progresista. En estos años, los discursos de la seguridad ciudadana no se han convertido, por diferentes factores, en elementos de política pública (Zavaleta Betancourt 2013).

En el debate hay una serie de estudios que han producido el giro antropológico. Detengámonos un poco en ellos, dado el carácter central de su contribución en el campo. En los debates de algunas redes académicas latinoamericanas -aunque también en muchas organizaciones civiles que intervienen en el campo de la violencia- la etnografía del estado (Azaola Garrido 2006; Abrams, Gupta, Mitchell 2015) y de los grupos urbanos de microtráfico, los análisis de extracción de renta múltiple por la delincuencia organizada, las autodefensas y los linchamientos, la descripción etnográfica del estado realmente existente, han tenido una recepción extraordinaria, debido a que se considera que la descripción tradicional del estado invisibiliza el carácter social de la violencia.

A mi juicio, el giro antropológico en nuestro campo contribuye a la comprensión de la dinámica de las violencias, describe de otro modo la densidad del sentido que los mexicanos y latinoamericanos damos al proceso, pero, más allá de la debilidad de algunas de sus evidencias, es débil en la explicación de estructuras sociales si no dialoga con la sociología y la ciencia política. En otro lugar he tratado acerca de las bases teóricas de los discursos de la antropología política quienes -reconozco- abren posibilidades de comprensión y explicación de la violencia pero utilizan parcialmente los discursos biopolíticos y de la sociología weberiana del Estado. En tal caso, la etnografía de lo estatal puede interpretarse no como una ruptura sino como extensión del discurso weberiano del Estado.

En esta lógica, hay un tercer acontecimiento discursivo en el campo, marginal aún, pero muy utilizado por diversos autores debido a sus fuertes implicaciones políticas. El análisis de la violencia como la renuncia al conflicto o bien de las consecuencia de políticas estatales extrajudiciales que suponen excepciones y estado de excepción (Arteaga Botello y Arzuaga Magnoni 2016; Pérez Correa et al. 2015; Illades y Santiago 2014). Estas perspectivas, en líneas generales, aún no producen análisis empíricos regionales, pero tienen gran aceptación en muchas publicaciones académicas.

Evidentemente, el campo de los estudios sobre la violencia es también un espacio en donde se han logrado otros acuerdos significativos, por ejemplo: se acepta la acumulación histórica de la violencia social y política (Misse en: Jaramillo 2014; González y González 2015), la violencia como un campo 
dinámico relacionado con los campos económicos y políticos (Wacquant 2001), la estructuración de una sociedad incivil y la acción colectiva de la delincuencia organizada, la policialización de los militares como un dispositivo de control, la centralidad del análisis de la violencia sociopolítica en la construcción de un régimen de baja intensidad democrática, los usos políticos de los discursos que trascienden la seguridad pública, entre otros. De este inventario quizá las ideas de Michel Misse (en: Jaramillo 2014) sean las más elocuentes:

La violencia parece, más bien, un proceso social que exige acumulación histórica, aunque el desarrollo de mercados ilícitos [...] pueda ser importante e incluso central en esta acumulación originaria y en sus resultados posteriores (Misse en: Jaramillo 2014: 59).

En estos años, las nuevas generaciones de investigadores hemos aprendido que la violencia social ni agota lo social ni lo destruye; produce saberes y sujetos, tiene una nueva "sociabilidad", asimismo, aceptamos que las prácticas de victimización dependen de posicionamientos móviles en la correlación de fuerzas, que el monopolio de la violencia legítima es una construcción que produce sus contrapartes ilegales (Agudo Sanchíz 2011), la incivilidad se relaciona con las diversas modalidades de lo ilícito, tolerado, no tolerado y lo capturado por la economía legal; la violencia de la delincuencia organizada es un instrumento para la reproducción de capitales ilegales, la economía legal incluye a la economía ilegal (Giraldo Ramírez et al. 2014); la etnografía del dolor y el sufrimiento no puede reducirse al periodismo presuntuoso que no se interroga acerca de las consecuencias prácticas del dolor en los circuitos de violencia incentivados por la venganza, el resentimiento y las inclusiones colaterales o ilegales en la modernidad del país (Ortega 2008).

\section{La impunidad y las reformas de las instituciones de seguridad y justicia}

La violencia social arriba descrita se reproduce en la impunidad. La debilidad de las instituciones de seguridad y justicia bajo reforma han contribuido a la reproducción de la violencia social. Estas circunstancias nos obligan a la observación de las reformas policiales y de la justicia penal en la medida en que abren las posibilidades de observación en nuestro campo de estudios. La observación externa -no orientada a los indicadores de desempeño- puede orientarnos en la comprensión de los obstáculos de la implementación y los retos de las reformas.

En México no se castigan el 92,8\% de los delitos, del 7,2\% restantes denunciados el 5,3\% no se investigan y el otro $1,9 \%$ terminan con sentencias condenatorias (INEGI 2015). De acuerdo con Carbonell (2016), el sistema de justicia penal se caracteriza por la falta de capacitación, la corrupción, las fallas de investigación; el inacabamiento de las leyes y del diseño institucional
El campo de los estudios de la violencia en México

José Alfredo Zavaleta Betancourt 
Dossier América Latina: violencia en México y Centroamérica de la procuración y administración de justicia y el abuso de la prisión preventiva, mientras los operadores de las reformas experimentan aún una curva de aprendizaje (Carbonell 2016).

La observación puede interrogarse acerca de los factores que bloquean la implementación de las reformas y cómo las resistencias al cambio forman parte de las prácticas que producen la impunidad en la cual se multiplica la violencia social.

La reforma de las instituciones de seguridad y justicia en nuestro país es otro episodio de la globalización de las reformas policiales y judiciales implementadas en las sociedades latinoamericanas. En nuestro caso, estas reformas han sido precedidas por la militarización de las policías -policialización del Ejército- y la guerra contra la delincuencia organizada. Estos procesos de reforma no han sido resultado de investigaciones científicas, diagnósticos, análisis de coyunturas como tampoco el insumo principal de los programas de prevención.

La militarización de las policías y la policialización de los militares en la guerra interna contra las organizaciones delictivas, han abierto una disputa en las instituciones policiales que ha producido tanto la coalición de algunos policías municipales, estatales y federales y las redes delictivas regionales en los cuales se implementan los operativos conjuntos del gobierno federal tanto como un patrón de letalidad y estados de excepción regionales en el país.

La reforma policial no ha logrado la conformación de una policía nacional y el mando único sigue debatiéndose como un mecanismo de centralización de bajas capacidades institucionales y corrupción sin contrapesos civiles efectivos debido a los gobiernos divididos y los cálculos de los partidos políticos dominantes en los gobiernos estatales y el gobierno federal. La militarización de la seguridad pública ha implicado efectos indeseables tales como la deserción militar y el financiamiento indirecto o no deseado de las organizaciones de la delincuencia organizada vía la deserción de sus efectivos. Decía Samuel Antonio González Ruíz:

La delincuencia organizada es el síntoma, no la enfermedad (la debilidad estatal) [...] Los policías municipales y algunos militares cuando desertan van a dar directamente a la delincuencia, es decir, que nosotros como país les pagamos el entrenamiento contribuyendo a la profesionalización del narcotráfico (citado en: Ortega 2011).

La vieja militarización de las policías ha continuado por otros medios, a pesar de que se decretó la implementación de un nuevo modelo de justicia penal orientada a la investigación de los delitos. La policialización de los militares -incluida la policía federal y la gendarmería- es un obstáculo a la implementación de un sistema policial en el marco de la reforma de la justicia penal. El Ejército, a pesar de aceptar las resoluciones judiciales federales en torno al fuero militar, es inexpugnable en casos de violación de derechos humanos. 
La implementación de reforma de la justicia penal ha sido sujeta a múltiples negociaciones, de tal forma que los avances de implementación son híbridos organizacionales que indican que las metas institucionales de la mediación, la descarga de los jueces, la reducción del encierro están lejos de constituir los elementos predominantes del sistema de justicia penal (Alvarado Mendoza 2015). En muchos casos -pero, sobre todo, en aquellos en los cuales policías y militares participaron en abusos de fuerza, desapariciones forzadas o ejecuciones extrajudiciales- durante la presunción de inocencia, se hace uso de amparos o se distorsionan pruebas. El sistema de justicia penal es por ahora un nuevo mercado de justicia en el cual el encierro es una forma de expulsión social. Dice Arturo Alvarado Mendoza:

[...] resultado de múltiples procesos de acuerdos y reformas legales, hoy día el 'sistema' de justicia es un enorme y complejo aparato normativo y burocrático, sin una lógica uniforme de operación, gobernado por criterios políticos del momento y no por un bien diseñado programa de acción [...] formalmente los nuevos regímenes penales son menos punitivos [...] porque limitan la capacidad punitiva del estado y han incluido sistemas pedagógicos $y$ de justicia alternativa, todavía existen límites a las garantías, violaciones al debido proceso, maltrato discriminación y erosión de los derechos de toda la población (Alvarado Mendoza 2014).

Por otra parte, de la misma forma que la reforma policial ha excluido las formas de participación comunitaria y autogestionarias, la reforma de la justicia penal ha implicado un proceso de la descentralización de la justicia penal sin el reconocimiento de otras legalidades, tales como los usos y costumbres indígenas, y las formas de autovigilancia y castigo de las comunidades, tales como los jueces indígenas y las policías comunitarias (Zavaleta Betancourt 2015; Poole 2006; Sierra, Hernández, Sieder 2013).

La reforma judicial neoliberal que se implementa en el país no ha sido analizada en su complejidad ni desde el prisma de la interlegalidad que produce el encuentro de una pluralidad de legalidades en los espacios regionales ni cómo se produce una paralegalidad de los grupos indígenas radicales, los citadinos y las organizaciones delictivas en los márgenes estatales. Asimismo, la reforma de la justicia penal estatal se ha focalizado en la capacitación genérica de los operadores de la procuración y administración de justicia y ha dejado el sistema penitenciario -muchos de ellos controlados mediante autogobiernos controlados por la delincuencia organizada- a la certificación global, la privatización de reclusorios federales y la capacitación de custodios mediante la institucionalización de la academia nacional penitenciaria.

Para el análisis de estos procesos, la nueva sociología latinoamericana y antropología política y jurídica mexicana nos dotan de elementos para la investigación de las instituciones estatales bajo reforma, tal como existen en las regiones en las cuales se producen excepciones, en los márgenes estatales,
El campo de los estudios de la violencia en México

José Alfredo Zavaleta Betancourt 
Dossier América Latina: violencia en México y Centroamérica como parte del campo de la violencia y el delito. Los estudios de los antropólogos políticos y jurídicos nos ayudan a repensar lo que hemos entendido hasta ahora como Estado mexicano, puesto que -lejos de las viejas conceptualizaciones de éste como desarrollista, social autoritario, populista-, lo caracterizan como una pluralidad de regímenes de la misma forma que las instituciones son presentadas como construidas imaginariamente por los funcionarios, burócratas y los ciudadanos.

El abandono del concepto del Estado como "cosa” o de su morfología como un aparato, posibilita la observación de su heterogeneidad y funciones respecto del nuevo modelo de desarrollo neoextractivista predominante en algunas regiones mexicanas. Los análisis regionales comparados de las reformas de la policía y la justicia penal no pueden separarse de la observación de las funciones de la delincuencia organizada en el despojo por coacción de las propiedades comunitarias concesionadas bajo el argumento de "interés público".

La antropología jurídica y política nos han enseñado que la institucionalización de las prácticas que denominamos Estado de derecho neoliberal es un mecanismo de la colonización de las consecuencias perversas de la desigualdad porque militarizan el control de los clientes políticos y eliminan a los sujetos peligrosos que produjeron mediante los mecanismos de la baja distribución y la exclusión, tanto como mediante la criminalización, el incremento de penas y las ejecuciones extrajudiciales.

En estas circunstancias podemos interrogarnos: ¿De qué hablamos cuándo nos referimos a las reformas de la policía y la justicia del Estado neoliberal mexicano? En general, en nuestro país se habla de la necesidad de una forma estatal de castigo del delito y la violencia que se focaliza en el proyecto del mando único y las capacidades institucionales de investigación de la policía para los juicios orales, tanto como de la reforma de la justicia penal como un mecanismo público de sentencia de los indiciados -en los grados asimétricos de avance de implementación en los estados- son hasta ahora, la integración organizacional de las excepciones y prácticas extrajudiciales y las reformas judiciales estatales un espacio para la impunidad institucionalizada tanto como el encierro penitenciario fabrica delincuentes.

\section{Los retos de la agenda sobre violencia social}

De la misma forma que los procesos de reforma institucional, el campo de la violencia y delito tienen una lógica distinta en las regiones del país. La agenda académica de las ciencias sociales mexicanas en el campo de la violencia puede orientarse en los próximos años al análisis de los márgenes estatales en el campo de la violencia y el delito y en la subjetividad de las víctimas: desaparecidos, familiares de desaparecidos, extorsionados, secuestrados, ejecutados, fosas clandestinas, asesinatos de periodistas, captura de policías locales, reformas inacabadas de seguridad y justicia, instituciones capturadas, vigilan- 
cia policiaca virtual y la información política ilegal mediante intervenciones telefónicas.

Particularmente, es importante consolidar los programas de investigación predominantes en el campo; desarrollar metodologías mixtas y estudios longitudinales; promover la integración de redes académicas regionales; incentivar la investigación acción; institucionalizar un código de ética; hacer un metaanálisis de los riesgos del trabajo de terreno; ir a fondo en el análisis de la heterogeneidad de las instituciones estatales tal como existen en los márgenes y las interfaces de las organizaciones delictivas y las redes políticas (RGVS 2015). Dice uno de nuestros reportes coordinado por A. Alvarado Mendoza:

1. Desarrollar proyectos en torno a las líneas anteriores. Hay temas de crimen tradicionales que deben seguirse estudiando como homicidios, violencia familiar, violencia del estado. Adicionalmente se deben promover estudios que aumenten el conocimiento en áreas que no conocemos como armas, desplazados, desaparecidos, trata, producción y efectos de las drogas ilícitas, sicariato, feminicidios. También la realización de estudios pilotos como los que se han hecho en estudios sobre despojo de tierras y bienes. Paralelamente, debemos promover la generación de teorías desde la experiencia nacional, como por ejemplo aquellas sobre el crimen organizado, que se puede estudiar y desarrollar desde la experiencia nacional.

2. Desarrollar estrategias metodológicas interdisciplinarias mixtas y estudios longitudinales.

3. Promover la integración de redes académicas regionales.

4. Apoyar y difundir aquellas investigaciones que incluyen propuesta de intervención para reducir la violencia.

5. Promover un código de ética común a estas investigaciones.

6. Hacer énfasis en la prevención de la violencia como política pública de acción. En este tema se propone que las políticas estén sustentadas en evidencias y en la mejora de las bases de datos. Así también promover trabajos sobre experiencias exitosas en reducción de la violencia que apunten a las resiliencia y pacificación.

7. Valorar el incremento de riesgos en el trabajo de campo y proponer medidas de protección.

8. Impulsar los estudios sobre las fuerzas coercitivas del Estado. ¿Qué está pasando con las fuerzas coercitivas del Estado? ¿Cómo ha evolucionado su papel en la intervención? ¿Se han hecho más violentas o menos? ¿Qué efecto tiene la violencia de las fuerzas en la sociedad y en la generación de nuevas violencias? Hay una clara transformación de las policías en los años recientes pero desconocemos las consecuencias. ¿Qué modelos funcionan en otras partes? ¿Cuáles no? ¿Cómo tratan las autoridades ciertos delitos, no sólo las penales, sino en general?

9. Estudios sobre organizaciones criminales ¿Cómo han evolucionado? ¿Cómo ha cambiado su forma de operar? ¿Cómo son sus prácticas de control? Narco cultura- cultura de la violencia en general. La delincuencia llamada orga-
El campo de los estudios de la violencia en México

José Alfredo Zavaleta Betancourt 
Dossier América Latina: violencia en México y Centroamérica nizada (principalmente el tráfico ilícito de drogas ilegales, pero también el tráfico de armas, el contrabando, la piratería, el tráfico humano y de órganos humanos, lavado de dinero, más la apreciable corrupción gubernamental y la violencia política, la inmensa economía ilegal internacional), los conflictos sociopolíticos históricos acumulados (post-conflicto, además de la violencia urbana u otros de alta presencia de bandas organizadas como maras, barras o pandillas violentas que parecen controlar territorios enteros y azorar, que no gobernar poblaciones enteras).

10. Interfaces entre organizaciones criminales y fuerzas coercitivas del estado u otras instituciones estatales. (RGVS 2015).

La observación empírica orientada teóricamente con reflexiones situadas en lo local podría focalizarse en el análisis mixto de la acumulación de la violencia en las regiones, particularmente en la violencia de la delincuencia organizada, mediante registros cuantitativos y cualitativos de la continuidad y discontinuidad de las formas de violencia política y social en el proceso de construcción del Estado de derecho neoliberal, en estados con alternancia y sin alternancia en las gubernaturas; la articulación de lo rural y urbano en las trayectorias sociales y delictivas de jóvenes, en transiciones biológicas y la decisión de participar en el campo de la violencia y el delito, sus entradas y salidas en el campo de lo ilícito y lo ilegal; las representaciones sociales, el dolor, el sufrimiento de las familias de víctimas y de victimarios aprehendidos o desaparecidos por la delincuencia organizada o las instituciones de seguridad; los regímenes de la producción de contrapartes ilegales por parte de las instituciones estatales para desechar a grupos de población inútiles para la reproducción de capitales globales; los obstáculos organizacionales y sociales de los procesos de reforma institucional.

En este proceso será imprescindible el diálogo reflexivo, crítico, efectivo con las asociaciones civiles regionales de defensoría de derechos y las élites locales tanto como la intervención profesional en las instituciones desde afuera. Debemos tomarnos en serio la crítica de autoreferencia y poco sentido práctico. Estos elementos de la agenda nos obligan a la auto-reflexión -muy desarrollada por cierto entre los antropólogos-acerca de los dilemas éticos del trabajo de investigación, por ejemplo, las implicaciones políticas del encargo, el uso de los datos, la gestión del miedo propio, la relación con las autoridades cuando se trabaja con los agentes del campo de la violencia y el delito.

\section{Conclusiones}

La observación de la violencia y el delito como un campo sociopolítico posibilita una descripción realista de las relaciones de un conjunto de agentes que intervienen dentro y fuera de las universidades públicas y privadas para reducir las tasas delictivas o para controlar los factores y efectos indeseables de estos procesos. 
El campo de estudios sobre la violencia en nuestro país se ha construido mediante la superación de un conjunto de prejuicios y teorías insuficientes que no posibilitan la comprensión de la lógica y sentido de la violencia social porque la consideran una forma de violencia política, o bien por no tematizarla como parte de sus modelos normativos. En efecto, la observación de la violencia social en México ha requerido de la superación de la teoría de la transición democrática y del socialcivilismo antipolítico que no considera la variable de la impunidad en las reformas de las instituciones de seguridad y justicia.

El campo de estudios de la violencia y el delito no se produce sólo en las universidades públicas, también en las organizaciones civiles y consultorías críticas del teoricismo de algunos ensayos académicos sobre el tema. De esa matriz discursiva proviene la idea inadecuada de la sobrediagnosticación de la violencia. Por lo contrario, de esta opinión tan generalizada entre consultores, debemos reconocer que sabemos poco acerca de las nuevas formas de la violencia social porque la consideramos una variable política ligada al régimen, por ejemplo sabemos muy poco acerca de la articulación de las violencias caciquil, contrainsurgente y social; en torno a la articulación de violencia en los espacios rurales y urbanos; sobre la relación entre violencia y delito; acerca de la violencia en las escuelas y acoso escolar y sexual; de las trayectorias sociales frágiles de jóvenes o bien de la corrupción institucional, la letalidad del uso de la fuerza por agentes gubernamentales y el tipo de guerra que se libra en nuestro país.

Para la observación de estos procesos, la divulgación de las ideas de autores clásicos es importante pero no suficiente; por lo contrario, el análisis regional, local, situado, que utiliza conceptos de teorías de sociedades centrales, no debe olvidar la historicidad de nuestras instituciones estatales y la desigualdad histórica que determina los procesos de violencia local. Por fortuna, el campo ha evolucionado mediante ciertos acontecimientos discursivos que han posibilitado la idea de victimización, seguridad ciudadana, la heterogeneidad de las instituciones de seguridad y justicia y el análisis cultural de la violencia urbana.

Particularmente, la observación de la violencia social desde una perspectiva cultural disputa un lugar en el debate público a los estudios sobre seguridad ciudadana. En las universidades y organizaciones civiles se habla cada vez más de violencia y en las cámaras empresariales e instituciones de seguridad ciudadana; pero independientemente de los diferentes posicionamientos, en el campo nacional, un sub-campo latinoamericano reconoce como logros el reconocimiento de que la violencia se acumula, puede conceptuarse como un campo, posibilita una sociedad incivil, incluye la militarización de policías y la policialización de los militares que ha producido la violación de derechos humanos y bloquea las reformas, y que en el campo existen usos liberales, neoliberales y progresistas de los derechos humanos.

La observación externa de las reformas de las instituciones de seguridad y justicia indican que son parte de la globalización de reformas precedidas por la militarización. Así, en nuestro país, en el campo de la violencia y el delito,
El campo de los estudios de la violencia en México

José Alfredo Zavaleta Betancourt 
Dossier América Latina: violencia en México y Centroamérica la militarización ha coexistido con la corrupción institucional, la prevención inútil, la centralización de mando y el incremento de la letalidad, la baja capacitación y debilidad del sistema de justicia penal. En estas circunstancias, la prevención ha sido un dispositivo de legitimación de la militarización, la reforma policial se reduce al control centralizado de mando y la reforma de la justicia penal se ha convertido en un híbrido institucional que no garantiza hasta ahora las metas de pacificación incluidas en los planes de desarrollo o de prevención, tampoco del mando único ni de reforma de la justicia penal.

\section{Bibliografía}

Abrams P., Gupta A., Mitchell T. (2015), Antropología del Estado, FCE, México.

Agudo Sanchíz A., Estrada Saavedra M. (eds.) (2011), Transformaciones del Estado en los márgenes de Latinoamérica, COLMEX-UIA, México.

Alexander J. C. (2012), Trauma. A Social Theory, Polity Press, Cambridge.

Alvarado Mendoza A. (2014), La reforma híbrida. Las transformaciones constitucionales a las instituciones de justicia y al Estado mexicano en la última década, "Letras Jurídicas", no 30, Universidad Veracruzana, México, disponible en: http://revistas.uv.mx/index.php/ letrasjuridicas/article/view/1521, fecha de consulta: 02.02.2016.

Alvarado Mendoza A., Arzt S. (eds.) (2001), El desafío democrático de México, COLMEX, México.

Angarita Cañas P. E. (comp.) (2015), Drogas, policía, delincuencia. Otras miradas a la seguridad ciudadana en América Latina, CLACSO, Argentina, disponible en: http://biblioteca.clacso. edu.ar/clacso/gt/20150626015959/Drogas.pdf, fecha de consulta: 02.02. 2016.

Arteaga Botello N., Arzuaga Magnoni J. (2016), Del neofuncionalismo a la conciencia icónica: ensayo crítico para pensar la sociología cultural de Jeffrey Alexander, "Sociológica", no 87, enero-abril, disponible en: http://www.revistasociologica.com.mx/pdf/8701.pdf, fecha de consulta: 06.07.2016.

Astorga L. (2015), ¿Qué querían que hiciera?, Grijalbo, México.

Azaola Garrido E. (2006), Imagen y autoimagen de la policía de la Ciudad de México, Ediciones Coyoacán, México.

Bailey J. (2014), Crimen e impunidad, Debate, México.

Bailey J., Godson R. (2000), Crimen organizado y gobernabilidad democrática, Grijalbo, México.

Bourdieu P. (2014), Sobre el Estado, Anagrama, Barcelona.

Bourgois P. (2010), En busca de respeto. Vendiendo crack en Harlem, Siglo XXI, Buenos Aires.

Buscaglia E. (2013), Vacíos de poder. Cómo combatir la delincuencia organizada, Debate, México.

Buscaglia E. (2015), Lavado de dinero y corrupción política. El arte de la delincuencia organizada internacional, Debate, México.

Calveiro P. (2012), Violencias de Estado. La guerra antiterrorista y la guerra contra el crimen como miedos de control global, Siglo XXI, Buenos Aires. 
Carbonell M. (2016), Nuevo sistema de justicia penal, entrevista en: "México Social", Canal Once, 09.02.2016, disponible en: https://www.youtube.com/watch?v=iXxdlfo57TM, fecha de consulta: 16.02.2016.

Dammert L., Bailey J. (coord.), Seguridad y reforma policial en las Américas: experiencias $y$ desafíos, Siglo XXI, México.

Das V., Poole D. (2008), El Estado y sus márgenes. Etnografías comparadas, "Cuadernos de Antropología Social", no 27, pp. 19-52.

De Souza Santos B. (2009), Sociología jurídica crítica. Para un nuevo sentido común en el derecho, Trotta-ILSA, Madrid.

Duque Daza J. (2015), Corrupción, organizaciones criminales y accountability. La apropiación de las regalías petroleras en los Llanos Orientales, Universidad del Valle, Cali.

Flores Pérez C. (2013), Historias de polvo y sangre. Génesis y evolución del tráfico de drogas en el estado de Tamaulipas, Ediciones La Casa Chata-CIESAS, México.

Fuentes Díaz A. (ed.) (2012), Necropolítica, violencia y excepción en América Latina, BUAP, Puebla.

Giraldo Ramírez J. et al. (coord.) (2014), Nuevas modalidades de captación de rentas ilegales en Medellín, EAFIT-ESU-Alcaldía de Medellín, Medellín.

González Rodríguez S. (2014), Campo de guerra, Anagrama, Barcelona.

González y González F. E. (2015), Poder y violencia en Colombia, Universidad Javeriana, Bogotá.

Henri-Lévy B. (1992), Las aventuras de la libertad, Anagrama, Barcelona.

Illades C., Santiago T. (2014), Estado de guerra. De la guerra sucia a la narcoguerra, Era, México.

INEGI, Instituto Nacional de Estadística y Geografía (2015), Encuesta Nacional de Victimización y Percepción sobre Seguridad Pública (ENVIPE), México.

Jaime E. (2016), Rendirse no es opción, “Excélsior”, 05.03.2016, disponible en: http://www. excelsior.com.mx/opinion/edna-jaime/2016/03/05/1078956, fecha de consulta: 15.03.2016.

Jaramillo A. M., Pera C. M. (eds.), Ciudades en la encrucijada, Violencia y poder criminal en Río de Janeiro, Medellín, Bogotá y Ciudad Juárez, IEPRI, IDRC, Bogotá.

Kalyvas S. N. (2010), La lógica de la violencia en la guerra civil, Akal, Madrid.

Kessler G. (2009), El sentimiento de inseguridad. Sociología del temor al delito, Siglo XXI, Buenos Aires.

Lemus J. J. (2015), Tierra sin Dios. Crónica del desgobierno y la guerra en Michoacán, Grijalbo, México.

Maerker D. (2011), "Nuestra guerra": Una conversación, "Nexos", 01.11.2011, disponible en: http://www.nexos.com.mx/?p=14554, fecha de consulta: 16.02.2016.

Maldonado S. (2010), Los márgenes del Estado mexicano. Territorios ilegales, desarrollo y violencia en Michoacán, Colegio de Michoacán, Zamora de Hidalgo.

Martínez Ó. (2010), Los migrantes que no importan. En el camino con los centroamericanos indocumentados en México, Icaria Editorial, Barcelona.

Mastrogiovanni F. (2016), Ni vivos ni muertos, La desaparición forzada en México como estrategia de terror, De bolsillo, México.
El campo de los estudios de la violencia en México

José Alfredo Zavaleta Betancourt 
Dossier América Latina: violencia en México y Centroamérica
México Evalúa (2015), Prevención de la violencia en México. ¿Cuáles son las prioridades?, México.

Misse M. (2014), Río de Janeiro: "sufrir la violencia, decir la paz", en: Ciudades en la encrucijada, Violencia y poder criminal en Río de Janeiro, Medellín, Bogotá y Ciudad Juárez, Jaramillo A. M., Pera C. M. (eds.), IEPRI, IDRC, Bogotá.

Moloeznik M. P. (2005), Seguridad pública y reforma policial en México. ¿Cambio o continuidad? en: Seguridad y reforma policial en las Américas: experiencias y desafíos, Dammert L., Bailey J. (coord.), Siglo XXI, México.

Ortega F. A. (ed.) (2008), Veena Das: sujetos del dolor, agentes de dignidad, Universidad Nacional de Colombia, Pontificia Universidad Javeriana, Instituto Pensar, Bogotá.

Ortega I. (2011), Narcotráfico, delincuencia organizada y violencia, síntomas de la debilidad del Estado, "Nexoveracruzano", disponible en: http://www.nexoveracruz.com/infonexo/estatales/ congreso/8597-narcotrafico-delincuencia-organizada-y-violencia-sintomas-de-la-debilidaddel-estado.html, fecha de consulta: 16.02.2016.

Pérez Correa C. et al. (2015), Índice de letalidad, menos enfrentamientos, más opacidad, "Nexos", 01.08.2015, disponible en: http://www.nexos.com.mx/?p=25468, fecha de consulta: 16.02.2016.

Poole D. (2006), Los usos de la costumbre. Hacia una antropología jurídica del Estado neoliberal, "Alteridades", vol. 15, no 31, enero-junio de 2006, pp. 9-21, disponible en: http://www.redalyc. org/pdf/747/74703102.pdf, fecha de consulta: 16.02.2016.

RGVS (2015), Reporte de Grupo de Violencia Social para el Consejo Consultivo de la Presidencia de la República y el CONACYT de México, coordinado por Arturo Alvarado Mendoza, en prensa.

Rico J. M., Chinchilla L. (2002), Seguridad ciudadana en América Latina, Siglo XXI, México, disponible en: http://biblioteca.udgvirtual.udg.mx/eureka/pudgvirtual/Seguridad\%20 ciudadana.pdf, fecha de consulta: 16.02.2016.

Roché S. (1996), La societé incivile. Qu’est-ce que l'insecurité?, Seuil, Paris.

Rodríguez Cuadros J. C. (2015), Génesis, actores y dinámicas de la violencia política en el Pacífico nariñense, Universidad Javeriana, Bogotá.

Schedler A. (2015), En la niebla de la guerra. Los ciudadanos ante la violencia criminal organizada, CIDE, México.

Sierra M. T., Hernández A. R., Sieder R. (eds.) (2013), Justicias indígenas y Estado. Violencias contemporáneas, FLACSO-CIESAS, México.

Wacquant L. (2001), Parias urbanos. Marginalidad en la ciudad en comienzos del milenio, Manantial, Buenos Aires.

Weber M. (1988), Sobre la teoría de las ciencias sociales, Premia, México.

Zavaleta Betancourt J. A. (2011), El campo de los delitos en México, "El Cotidiano", no 170, pp. 15-25.

Zavaleta Betancourt J. A. (coord.) (2013), La inseguridad y la seguridad ciudadana en América Latina, CLACSO, Buenos Aires, disponible en: http://www.biblioteca.clacso.edu.ar/clacso/ gt/20121123043123/Lainseguridadylaseguridadciudadana.pdf, fecha de consulta: 16.02.2016. 